Julia Hugo

\title{
Eltern und Schule - die Rolle von Eltern im Schulverhältnis als Rechtsverhältnis
}

\section{Zusammenfassung \\ Elternklagen führen vermehrt zu Rechtsunsicherheit auf Seiten der Lehrkräfte. Der Beitrag nähert sich daher Inhalt und Reichweite der Elternrolle im Schulverhältnis aus rechtlicher Perspektive an: zum einen historisch vor dem Hintergrund des Gesetzesvorbehalts im Schulverhältnis, zum anderen mit Blick auf die Schulgesetze der Länder, insbesondere Bayerns. Davon ausgehend werden Grenzen elterlicher Rechte thematisiert. Es zeigt sich, dass eine vertrauensvolle Zusammenarbeit von Eltern und Schule grundlegend für die gemeinsame Erziehungsaufgabe ist. \\ Schlüsselwörter: Schulrecht, Elternrechte, Schulverhältnis, Bildungs- und Erziehungsauf- trag \\ Parents and Schools - The Role of Parents in Their Relationship to the School as a Legal Relationship}

\begin{abstract}
Parental complaints often lead to legal uncertainty on the part of teachers. The article therefore approaches the content and scope of parental rights in school from a legal perspective: on the one hand, historically regarding the role of the legal reservation in school, on the other hand, with a view to the school laws of the federal states, especially Bavaria. On this basis, the limits of parental rights are discussed. It turns out that a trustful cooperation between parents and school is fundamental to the shared educational task.
\end{abstract}

Keywords: school law, parental rights, school relationship, educational mandate

Die Klagebereitschaft von Eltern ${ }^{1}$ gegen schulische Maßnahmen ist in den letzten Jahren v.a. im Zusammenhang mit Prüfungs- oder Versetzungsentscheidungen

1 Der rechtliche Begriff Eltern bezeichnet die Erziehungsberechtigten, d.h., v.a. „Personen, denen die Personensorge für das Kind oder den Jugendlichen zusteht" (Rux, 2018, Rn. 1095). 
gestiegen (BLLV, 2019). Medial wird eine starke Position der Elternschaft und eine defensive Rolle der Lehrkräfte propagiert (Dietrich, 2017). Jurist*innen betonen einerseits wiederholt die Notwendigkeit der konstruktiven Zusammenarbeit von Eltern und Schule (z.B. Böhm, 2019). Andererseits steigt die Zahl der SchulrechtsAnwält*innen mit einer auf das Elternklientel zugeschnittenen Expertise. Dies führt zu Rechtsunsicherheit bei Lehrkräften (BSZ, 2017). Der Beitrag zielt daher auf eine rechtliche Einordnung der Elternrolle im Schulverhältnis. Dafür wird diese erstens historisch mit Blick auf den Einzug des Gesetzesvorbehalts in den Schulbereich, zweitens ausgehend von Landesschulgesetzen und drittens konkret für das Bundesland Bayern skizziert (1). Schließlich erfolgt eine rechtliche Einordnung dieser Entwicklung i.H.a. Reichweite und Grenzen von Elternrechten (2).

\section{Rolle der Eltern im Schulverhältnis ${ }^{2}$}

Die Bereitschaft von Eltern, die Rechte ihrer Kinder im schulischen Kontext auch gerichtlich durchzusetzen, ist nicht nur Ausdruck eines neuen Selbstbewusstseins der Elternschaft gegenüber der Institution Schule oder des gesunkenen Ansehens des Lehrberufs (Mayr, 2016; statista, 2012). Sie fußt ferner strukturell auf rechtlichen Vorgaben, die die Berücksichtigung des Elternwillens in schulischen Angelegenheiten notwendig machen.

\section{Einzug des Gesetzesvorbehalts ${ }^{3}$ in das Schulrecht}

Bis in die 1970er-Jahre wurden in der BRD Eingriffe in die Grundrechte von Schüler*innen (z.B. körperliche Züchtigung als Erziehungsmaßnahme) über die Rechtsfigur des Schulverhältnisses als besonderes Gewaltverhältnis legitimiert und waren ohne gesetzliche Grundlage zulässig. Das änderte sich mit der Übertragung des Strafvollzugsbeschlusses des Bundesverfassungsgerichts (BVerfGE 33, 1-18) auf die Schule (Avenarius, 2019a, S. 327): Der Gesetzesvorbehalt als konstitutives Element demokratischer Rechtsstaatlichkeit müsse auch für die Schule gelten. Somit ist jeder wesentliche Eingriff in die Rechte von Kindern im Schulverhältnis als öffentlichem Rechtsverhältnis gesetzlich zu regeln. Vergleichbares gilt für Eltern: Zum einen tragen sie als gesetzliche Vertreter*innen für die Pflege und Erziehung ihrer Kinder die elterliche Sorge (\$1626 BGB) - auch im Kontext Schule. Zum anderen sind sie selbst gegenüber der Schule Grundrechtsträger*innen, v.a. aus Art. 6 Abs. 2 GG, demzufolge die Pflege und Erziehung der Kinder das „natürliche Recht“ der Eltern ist. Somit unterliegen auch Grundrechtseingriffe bei Eltern dem Gesetzesvorbehalt.

2 „Das Schulverhältnis [...] umfasst die Gesamtheit der rechtlichen Beziehungen zwischen der Schule einerseits, dem Schüler (und seinen Eltern) andererseits" (Avenarius, 2019a, S. 327).

3 Der Vorbehalt des Gesetzes verweist auf die Möglichkeit, Grundrechte durch Gesetz oder aufgrund eines Gesetzes einzuschränken (Weber, 2019, S. 628). 
| Julia Hugo

\section{Schulgesetze der Bundesländer}

Rechte und Pflichten von Eltern als Träger*innen der elterlichen Sorge einerseits und als Grundrechtsträger ${ }^{\star}$ innen andererseits werden gesetzlich in den Landesschulgesetzen $^{4}$ geregelt (vgl. Tab. 1).

Tab. 1: Regelungen mit Eltern-Bezug in den Schulgesetzen der Bundesländer.

\begin{tabular}{|c|c|}
\hline Bundesland & Zentrale Vorschriften der Landesschulgesetze \\
\hline Baden-Württemberg & §§ 55-61, 83, 85 SchG B-W \\
\hline Bayern & Art. $64-68,74,76$ BayEUG \\
\hline Berlin & $\S \S 88-91$ SchulG \\
\hline Brandenburg & §§ 81-82 BbgSchulG \\
\hline Bremen & §§ 6-6a, 60, 61 BremSchulG \\
\hline Hamburg & §§ $32,42,68-75,81 \mathrm{HmbSG}$ \\
\hline Hessen & §§ 72, 100-120, 154 SchulG HE \\
\hline Mecklenburg-Vorpommern & $\S \S 49,55,55 a, 86-90,92$ SchulG M-V \\
\hline Niedersachsen & $\S \S 55,71,88-100,169$ NSchG \\
\hline Nordrhein-Westfalen & $\S \S 120,123$ NRW - SchulG \\
\hline Rheinland-Pfalz & $\S \S 2,4,37-47,65,84$ SchulG \\
\hline Saarland & $\S \S 20 f ., 36$ SchoG \\
\hline Sachsen & §§ 45-50a SächsSchulG \\
\hline Sachsen-Anhalt & §§ 43, 55-63, 76 SchulG LSA \\
\hline Schleswig-Holstein & §§ 31, 69-78, 98 SchulG \\
\hline Thüringen & §§ 23, 31-32 ThürSchulG \\
\hline
\end{tabular}

Anm.: Es wurden Vorschriften ausgewählt, die schon in ihrer Überschrift Eltern, Erziehungsberechtigte oder Sorgeberechtigte als Regelungsadressat ${ }^{\star}$ innen explizit nennen. Für Teilabschnitte wurden alle Vorschriften des jeweiligen Abschnitts zitiert.

Quelle: eigene Darstellung

Jenseits der Normen mit explizitem Eltern-Bezug schon in der Überschrift (vgl. Tab. 1) werden Eltern auch in den übrigen schulgesetzlichen Regelungen berücksichtigt, etwa bei der Schulwahl. Da die Regelungen von Land zu Land variieren, folgt eine exemplarische Darstellung für das Bundesland Bayern. Im Übrigen sei auf das jeweilige Landesrecht verwiesen.

4 Eine Übersicht über alle Schulgesetze findet sich in der entsprechenden Datenbank der Kultusministerkonferenz (KMK, 2020). 
Bayerisches Gesetz über das Erziehungs- und Unterrichtswesen (BayEUG)

Der Elternrolle wird im Bayerischen Gesetz über das Erziehungs- und Unterrichtswesen $\left(B a y E U G^{5}\right)$ an mehreren Stellen Rechnung getragen: So ist das elterliche Erziehungsrecht nach Art. 1 Abs. 2 bei der Erfüllung des staatlichen Bildungs- und Erziehungsauftrags $\mathrm{zu}$ achten. Schule und Eltern erfüllen ferner eine gemeinsame Erziehungsaufgabe, die eine vertrauensvolle Zusammenarbeit erfordert (Art. 74 Abs. 1). Entsprechendes gilt für das Zusammenwirken von Lehrkräften und Eltern (Art. 59 Abs. 3). Ferner sind Eltern neben Schulleitenden, Lehrkräften und Schüle$\mathrm{r}^{\star}$ innen Teil der Schulgemeinschaft (Art. 2 Abs. 4 Satz 1).

Eltern haben insbesondere folgende „Verhaltenspflichten“ (Avenarius, 2019a, S. 328):

- Schulanmeldung (Art. 35 Abs. 4 Satz 1);

- Unterstützung der Erziehungsarbeit der Schule (Art. 76 Satz 1);

- Einhaltung schulischer Pflichten ${ }^{6}$ durch ihre Kinder (Art. 76 Satz 1), v.a. Teilnahme an Unterricht und Schulveranstaltungen (Art. 76 Satz 2);

- Beschaffung nicht lernmittelfreier Lernmittel (Art. 51 Abs. 4 Satz 1).

Das elterliche Recht zur Bestimmung des Bildungswegs (Avenarius, 2019a, S. $336 \mathrm{ff}$. umfasst v. a. Folgendes:

- Wahl der Pflichtschule bei mehreren Sprengelschulen mit unterschiedlichem Bildungsangebot (Art. 42 Abs. 1 Satz 2);

- Beantragung des Besuchs einer Grund- oder Mittelschule in einem anderen Sprengel aus zwingenden persönlichen Gründen (Art. 43 Abs. 1 Satz 1);

- Wahl des schulischen Bildungswegs (jenseits des Pflichtschulbereichs) (Art. 44 Abs. 1 Satz 1);

- Anmeldung zu Wahlfächern (Art. 50 Abs. 2 Satz 3);

- Beantragung von Notenschutz (Art. 52 Abs. 5 Satz 2 Nr. 4).

Glauben und Weltanschauung der Eltern werden z.B. in folgenden Punkten berücksichtigt:

- Untersagung des Tragens nicht wertneutraler Symbole (z. B. Kleidung) für Lehrkräfte (Art. 59 Abs. 2 Satz 3);

- Abmeldung vom Religionsunterricht (Art. 46 Abs. 4 Satz 1);

- Zustimmung zur Zuweisung zu Klassen mit gleichem Bekenntnis bei der Bildung von Parallelklassen an Grund- oder Mittelschulen (Art. 49 Abs. 2 Satz 2).

5 Alle nachfolgenden Normen sind, wo nicht anders vermerkt, dem BayEUG entnommen.

6 Für eine Übersicht aller schulischen Pflichten vgl. Hanschmann (2019a, S. 454 ff.). 
| Julia Hugo

Informations- und Auskunftsrechte der Eltern bzw. -pflichten der Schule betreffen z. B. dieses:

- Ziel, Inhalt und Form des Familien- und Sexualkundeunterrichts (Art. 48 Abs. 3);

- Notenbekanntgabe (Art. 52 Abs. 2 Satz 4);

- frühzeitige schriftliche Mitteilung über alle wesentlichen Vorgänge (z. B. Absinken des Leistungsstands) und Beratungsangebot im Fall des Nicht-Vorrückens (Art. 75 Abs. 1 u. 2);

- allgemeine Beratungsaufgaben (Art. 78 Abs. 1 Satz 1).

Mitwirkungsrechte der Eltern realisieren sich insbesondere im Elternbeirat (Art. 6468) und im Schulforum als gemischtem Gremium (Art. 69). Der Elternbeirat hat z.B. bei Ordnungsmaßnahmen nach Artikel 87 auf Antrag der Eltern oder der Schüler*innen ein Anhörungsrecht (Art. 88 Abs. 3 Satz 2 Nr. 3).

\section{Grenzen der Elternrechte}

Zusammengefasst kommt den Erziehungsberechtigten als Träger*innen eigener Grundrechte sowie als gesetzlichen Vertreter ${ }^{\star}$ innen ihrer Kinder eine gewichtige Rolle im Schulverhältnis zu. So werden in den Landesschulgesetzen - wie beispielhaft am BayEUG ausgeführt - die elterlichen Informations- und Auskunftsrechte sowie Entscheidungs- und Antragsrechte, sensible Erziehungsbereiche (z.B. Sexualkundeunterricht), die freie Berufswahl (z.B. Nicht-Versetzung) oder das allgemeine Persönlichkeitsrecht der Schüler*innen (z.B. Wahlfächer) geregelt. Auch wirken Eltern als Teil der Schulgemeinschaft in Gremien mit.

Diesen umfassend anmutenden Elternrechten sind jedoch Grenzen gesetzt: Dazu zählen die bereits angeführten Elternpflichten, die Grundrechte der Kinder selbst, der fiduziarische Charakter des elterlichen Erziehungsrechts (Art. 6 Abs. 2 GG), das stets im Sinne der Kinder auszuüben ist, sowie die dezidiert gemeinsame, durch den staatlichen Bildungs- und Erziehungsauftrag (Art. 7 Abs. 1 GG) bedingte Erziehungsaufgabe von Schule und Elternhaus. Dabei gibt es Bereiche, die allein der Staat verantwortet (Organisation des Schulwesens nach Schularten und -stufen; Ausgestaltung des Berechtigungswesens; vgl. Avenarius, 2019a, S. 335). In allen anderen Bereichen ist eine vertrauensvolle Zusammenarbeit von Eltern und Schule nötig, worauf Rechtsprechung (BVerfGE 34, 165) und Gesetzgebung (z. B. Art. 74 Abs. 1 Satz 1 BayEUG) immer wieder verweisen. ${ }^{7}$

Für den Ausnahmefall nicht vermittelbarer Differenzen stehen Eltern formlose (z.B. Aufsichtsbeschwerde) und förmliche Rechtsbehelfe (z.B. Widerspruch) zur

7 Zur Betonung der gemeinsamen Erziehungsaufgabe vgl. schon KMK (2003). 
Verfügung. ${ }^{8}$ Klagemöglichkeiten ergeben sich jedoch v. a. gegen Verwaltungsakte (z.B. Ordnungsmaßnahmen) und strafbare Handlungen der Lehrkraft (z. B. körperliche Gewalt). Dabei ist der Klagegegner auf verwaltungsrechtlicher Ebene immer das jeweilige Bundesland, nicht jedoch die Lehrperson. Einzelne fachliche, pädagogischdidaktische oder methodische Entscheidungen der Lehrkraft im Rahmen der unterrichtlichen und erzieherischen Tätigkeit sind von der pädagogischen Freiheit, einer weiteren Schranke des Elternrechts, umfasst (Avenarius, 2019b, S. 666 ff.).

Wenngleich die Rechtsprechung in den letzten Jahren einen Zuwachs an Elternklagen verzeichnet, Elternrechten im Schulrecht eine starke Position zukommt und sich Eltern vielfältige Rechtsschutzmöglichkeiten bieten, sind Eltern ein Teil der Schulgemeinschaft. Dem Elternwillen stehen mit dem eigenständigen staatlichen Bildungs- und Erziehungsauftrag und der pädagogischen Freiheit der Lehrkräfte zwei Pendants gegenüber, die die Reichweite des Elternrechts begrenzen und eine konstruktive Zusammenarbeit mit Blick auf die gemeinsame Erziehungsaufgabe erfordern.

\section{Literatur und Internetquellen}

Avenarius, H. (2019a). Schulverhältnis, Rechtsstellung der Schülerinnen und Schüler, Elternrecht. In H. Avenarius \& F. Hanschmann (Hrsg.), Schulrecht. Ein Handbuch für Praxis, Rechtsprechung und Wissenschaft (9., neubearb. Aufl.) (S. 327-343). Köln: Carl Link.

Avenarius, H. (2019b). Nichtvermögenswerte Rechte der Lehrkräfte. In H. Avenarius \& F. Hanschmann (Hrsg.), Schulrecht. Ein Handbuch für Praxis, Rechtsprechung und Wissenschaft (9., neubearb. Aufl.) (S. 637-670). Köln: Carl Link.

BLLV (Bayerischer Lehrerinnen- und Lehrerverband). (2019). Eltern und Lehrer: Gemeinsam fördern statt gegeneinander klagen. Zugriff am 02.06.2021. Verfügbar unter: https://www.bllv.de/vollstaendiger-artikel/news/eltern-und-lehrer-gemeinsam-foer dern-statt-gegeneinander-klagen/.

Böhm, T. (2019). Diese Note akzeptieren wir nicht - Welche Rechte Eltern in der Schule haben. München: mvg.

BSZ (Bayerische Staatszeitung). (2017). „Schwierige Schüler haben meist schwierige Eltern“. Zugriff am 02.06.2021. Verfügbar unter: https://www.bayerische-staatszeitung.de/ staatszeitung/politik/detailansicht-politik/artikel/schwierige-schueler-haben-meistschwierige-eltern.html\#topPosition.

Dietrich, A. (2017). Eltern gegen Lehrer. DIE ZEIT, 27. Zugriff am 02.06.2021. Verfügbar unter: https://www.zeit.de/2017/27/schule-eltern-lehrer-rechtsstreit-schulnoten.

Hanschmann, F. (2019a). Verhalten der Schülerinnen und Schüler. In H. Avenarius \& F. Hanschmann (Hrsg.), Schulrecht. Ein Handbuch für Praxis, Rechtsprechung und Wissenschaft (9., neubearb. Aufl.) (S. 454-475). Köln: Carl Link.

Hanschmann, F. (2019b). Rechtsschutz von Schülerinnen und Schülern sowie Eltern. In H. Avenarius \& F. Hanschmann (Hrsg.), Schulrecht. Ein Handbuch für Praxis, Rechtsprechung und Wissenschaft (9., neubearb. Aufl.) (S. 549-570). Köln: Carl Link.

KMK (Sekretariat der Ständigen Konferenz der Kultusminister der Länder in der Bundesrepublik Deutschland). (2003). Bonner Erklärung. Gemeinsame Erziehungs-

8 Zur Übersicht über den Rechtsschutz im Schulwesen vgl. Hanschmann (2019b). 
| Julia Hugo

verantwortung in Schule und Elternhaus stärken. Zugriff am 10.06.2021. Verfügbar unter: https://www.kmk.org/fileadmin/Dateien/pdf/PresseUndAktuelles/2003/bonner_20 erkl_E4rung.pdf.

KMK (Sekretariat der Ständigen Konferenz der Kultusminister der Länder in der Bundesrepublik Deutschland). (2020). Schulgesetze der Länder in der Bundesrepublik Deutschland. Stand: Dezember 2020. Zugriff am 02.06.2021. Verfügbar unter: https:// www.kmk.org/dokumentation-statistik/rechtsvorschriften-lehrplaene/uebersicht-schul gesetze.html.

Mayr, J. (2016). Lehrerpersönlichkeit. In M. Rothland (Hrsg.), Beruf Lehrer/Lehrerin. Ein Studienbuch (S. 87-102). Münster: Waxmann.

Rux, J. (2018). Schulrecht (6., vollst. neubearb. Aufl.). München: Beck.

statista. (2012). Umfrage in Deutschland zum Lehrerberuf. Zugriff am 02.06.2021. Verfügbar unter: https://de.statista.com/statistik/daten/studie/241781/umfrage/umfrage-in-deut schland-zum-lehrerberuf/\#professional.

Weber, K. (2019). Gesetzesvorbehalt. In C. Creifelds ( $\dagger$ ) \& K. Weber (Hrsg.), Rechtswörterbuch (23., neubearb. Aufl.) (S. 628). München: Beck.

Julia Hugo, geb. 1989, Wissenschaftliche Assistentin am Lehrstuhl für Schulpädagogik sowie Doktorandin am Lehrstuhl für Allgemeine Pädagogik der Ludwig-MaximiliansUniversität München (LMU).

E-Mail: julia.hugo@campus.lmu.de

Korrespondenzadresse: Ludwig-Maximilians-Universität München (LMU), Leopoldstr. 13, 80802 München 